A. Weisbecker, M. Burmester \& A. Schmidt (Hrsg.): Mensch und Computer 2015 Workshopband, Stuttgart: Oldenbourg Wissenschaftsverlag, 2015, S. 491-498.

\title{
Development and Evaluation of a Virtual Reality Driving Simulator
}

\author{
Quinate Chioma Ihemedu-Steinke ${ }^{1,2}$, Demet Sirim ${ }^{1}$, Rainer Erbach ${ }^{1}$, \\ Prashanth Halady ${ }^{1}$, Gerrit Meixner ${ }^{2}$ \\ Center of Competence HMI Leonberg, Robert Bosch $\mathrm{GmbH}^{1}$ \\ UniTyLab, Heilbronn University ${ }^{2}$
}

\begin{abstract}
This work presents the design and set-up of a virtual reality driving simulator for development and evaluation of future automotive HMI concepts. The Oculus Rift based virtual reality driving simulation described in this work is an immersive 3D virtual reality simulator, which gives the driver a near-realistic driving experience. Oculus Rift is a low-latency $360^{\circ}$ head tracking virtual reality head-mounted-display which offers a fully immersive 3D experience in virtual environments. It was developed in Unity3D engine and tested in a user experience evaluation in order to determine its level of immersion and the possible use of that technology for the development of future automotive HMI concepts.
\end{abstract}

\section{Introduction}

How would a driver react if faced with a hazardous situation? When is it best to alert the driver about unforeseen danger? What is the best way to interact with the driver in order to achieve the desired reaction? The above questions can be answered by analyzing the driver's behavior within a driving situation taking into account other drivers, the road conditions and the car dynamics. This is important since human error is one of the principal influencing factors that lead to road accidents, and is attributed to increased mental workload induced by distractions (Christodoulou et al., 2013). Placing the driver into a real driving situation in order to evaluate his behaviour is too dangerous whereas testing environments such as crash test environments are very time and cost intensive. Therefore, driving simulator (DS) is commonly used for research studies in monitoring the driver's behavior and for design and evaluation purposes of new HMI concepts. Mostly 2D-driving simulation environments are used in the automotive industry and for automotive research (De Winter et al., 2012; Krueger, 2005). These conventional DSs often lack the immersion of the driver into the driving scenario, and hence do not reveal the natural reaction and interaction. 
Emerging technologies in the field of virtual reality (VR) from the area of consumer electronics and increasing processing power offer potentials for new highly immersive driving simulation concepts e.g. in the development and evaluation of new advanced driver assistance systems (ADAS) (Bayarri et al., 1996; Cremer et al., 1996). This work presents first the benchmarking of the available state of the art tools for the development of an immersive VR DS in order to allow flexible, user-oriented adaptation of functions and HMI components in the context of a recursive, user-centered development for enhanced user experience. This approach is meant to compensate for the lack of total immersion and intuitive reaction of the test driver of conventional 2D DSs necessary for an effective analysis of a particular driving situation. Then the developed prototype is presented followed by a first evaluation with 25 users. Finally we discuss the results and give an outlook on further work.

\section{Current State of the Technology}

The level of immersion and hence the performance of a VR DS depends on the efficiency of the applied components and tools. Therefore, the most advanced components and tools were analyzed and benchmarked. Primarily, the performance of the system depends on:

1. Graphical Processing Unit,

2. Virtual Reality Devices,

3. Driving Simulator Tools and

4. External Interaction Devices.

\subsection{Graphical Processing Unit}

A literature review of suitable graphic cards for 3D gaming was performed based on the following core features; processing power, refreshment rate, pixel resolution, rendering technologies and supported APIs. Based on these criteria and available graphic cards benchmarking online as of the time this research was performed, Nvidia GeForce GTX 980M was selected (TopTenReviews, 2015; FurryBall 4.8 GPU Benchmark, 2014).

\subsection{Virtual Reality Devices}

The following devices were selected for the benchmark based on their usability and available information especially device specifications; OR DK2, Sony Morpheus, Samsung GearVR, Vuzix Wrap 1200DX-VR, Vrvana, GameFace, Cmoar, Sulon Cortex, InfinitEye, Antvr and Vrelia VReye. The analysis of the selected devices was performed according to the features listed on table 1. The next step involved the analysis of the contents to filter, and mark the key aspects and qualities of the HMD with respect to the level of immersion in the virtual environment (VE). Scores from 1 to 5 were allocated based on the following scaling; $5=$ very good, $4=$ good, $3=$ average, $2=$ not good and $1=$ poor or unknown. For example, Image refresh rates are considered faster in OLED than LCD, all devices with OLED display scored 5 as compared to 4 for LCD. Devices which offered more than $110^{\circ}$ field of view (FOV) 
scored 5 while those like OR with a maximum of $110^{\circ}$ FOV scored 4 . All selected devices offer a stereoscopic 3D view, so they all scored 5 in this category. OR DK2 was chosen as the most suitable device to develop the VR DS because of its low-persistence, low-latency, accurate positional tracking, and its large user community for FAQ

\begin{tabular}{|c|c|c|c|c|c|c|c|c|c|c|c|}
\hline Feature $\quad$ Device & $\begin{array}{c}\text { Oculus } \\
\text { DK2 }\end{array}$ & $\begin{array}{c}\text { Sony } \\
\text { Morpheus }\end{array}$ & $\begin{array}{l}\text { Samsung } \\
\text { Gear VR }\end{array}$ & $\begin{array}{l}\text { Vuzix Wrap } \\
\text { 1200DX-VR }\end{array}$ & Vrvana & GameFace & Cmoar & $\begin{array}{l}\text { Sulon } \\
\text { Cortex }\end{array}$ & InfinitEye & Antvr & $\begin{array}{l}\text { Vrelia } \\
\text { VReye }\end{array}$ \\
\hline Display Resolution & 5 & 5 & 5 & 4 & 5 & 5 & 4 & 4 & 5 & 5 & 5 \\
\hline Display Refreshness & 4 & 4 & 4 & 4 & 4 & 4 & 4 & 4 & 4 & 4 & 4 \\
\hline Display Type (OLED, LCD) & 5 & 3 & 5 & 3 & 5 & 3 & 4 & 4 & 4 & 4 & 4 \\
\hline Viewing Optics (FOV $\left.{ }^{*}\right)$ & 3 & 3 & 3 & 2 & 3 & 3 & 3 & 3 & 5 & 3 & 4 \\
\hline Interface HDMI & 5 & 5 & 1 & 5 & 5 & 5 & 1 & 1 & 5 & 5 & 5 \\
\hline Interface USB & 5 & 5 & 4 & 5 & 5 & 5 & 4 & 4 & 5 & 1 & 1 \\
\hline Internal Tracking Sensor & 5 & 4 & 5 & 5 & 5 & 5 & 3 & 5 & 5 & 5 & 3 \\
\hline Position Tracking Sensor & 4 & 4 & 5 & 5 & 5 & 5 & 4 & 5 & 5 & 5 & 4 \\
\hline Engine Integration tools (Unre & 5 & 2 & 1 & 1 & 5 & 4 & 3 & 1 & 1 & 1 & 4 \\
\hline SDK & 5 & 2 & 1 & 5 & 4 & 5 & 1 & 3 & 2 & 4 & 4 \\
\hline 3D Immersive Quality & 4 & 4 & 4 & 4 & 4 & 4 & 4 & 4 & 4 & 4 & 4 \\
\hline Active User Community & 5 & 3 & 4 & 4 & 3 & 4 & 3 & 3 & 3 & 3 & 3 \\
\hline Latency & 5 & 4 & 3 & 4 & 4 & 5 & 3 & 3 & 3 & 3 & 3 \\
\hline Persistence & 4 & 3 & 4 & 3 & 4 & 4 & 3 & 3 & 3 & 3 & 3 \\
\hline Steroscopic 3D & 4 & 4 & 4 & 4 & 4 & 4 & 4 & 4 & 4 & 4 & 4 \\
\hline \multirow[t]{2}{*}{ Weight } & 3 & 3 & 3 & 5 & 3 & 3 & 3 & 3 & 4 & 4 & 3 \\
\hline & $88,8 \%$ & $72,5 \%$ & $70,0 \%$ & $78,8 \%$ & $85,0 \%$ & $85,0 \%$ & $63,8 \%$ & $67,5 \%$ & $77,5 \%$ & $72,5 \%$ & $72,5 \%$ \\
\hline
\end{tabular}

Table 1: A Tabular representation of the State of the Art VR Device Rating

\subsection{Driving Simulator Tools}

SILAB was initially chosen for the evaluation of the VR DS with OR. However, an extra software package with a licence fee is required for integration with OR. Therefore, a benchmark was carried out in order to choose an alternative tool for the prototype development. Two renowned DS suppliers; SILAB and CarMaker, one open source tool; Open Scence Graph, and two renowned 3D gaming engines; Unreal Engine 4 and Unity3D were selected. The selected criteria for the benchmark were based on the tasks involved in developing a near-realistic and fully immersive 3D VR application and OR integration. Comprehensive information was collected from various sources including scientific publications, online sources, vendor websites, user forums as well as simulation books (Robinson, 2004). This benchmark was performed in five different steps namely; establishment of the benchmark requirements, survey and shortlisting of the software simulation tools, establishment of the evaluation criteria, evaluation of the software in relation to the criteria and software selection. Since some of these criteria for evaluation were dynamically changing during this thesis, iteration steps were required (Robinson, 2004). Unity3D was chosen because it offers a large Oculus user community for FAQ and a free version which provided all available features for the prototype implementation.

\subsection{External Interaction Devices}

Interactive devices used in this work are the Logitech G25 force feedback device bolted with steering input, gas-, brake- and clutch pedal and Leap Motion. The interaction is realized in Unity3D with the help of Logitech and Leap Motion plugins and core assets. 


\section{Material}

\subsection{Hardware}

Visualization Component: This consisted of the OR HMD, a Samsung monitor and the MSI computer monitor. The visualization components interact with the simulation through the Oculus SDK.

Input/Output Devices: The most important devices considered for this work are the Logitech G25, Leap Motion tracking device and solid 5.1 surround sound Roccat KAVE Gaming earphones. The control flow between the Logitech device and the 3D car model is realized in Unity3D with the help of Logitech plugins and assets (Logitech Gaming SDK, 2014). The Leap Motion served as a hand-and-finger-tracking device.

\subsection{Software}

Oculus SDK: Oculus SDK provides the library containing all the Prefabs necessary to create and run an application in OR e.g. the OVRPlayerController and OVRCameraRig, and the OculusUnityIntegration Package needed to integrate a Unity3D application with OR. Furthermore, all the necessary plugins needed to create and execute an application in oculus are contained in this library.

3D Models (3rd Party): The following 3D models were used in the development of the VR driving simulator; 3D BMW 7 Series model, V8 engine Soundpack -.fbx file, Landscape / Racetrack - .fbx files (Sommer, 2012), and Mixamo Carl Hi-Res / Male Character Pack (Mixamo, 2014). These models were either gotten from the Unity Asset store or bought directly from the $3 \mathrm{D}$ model supplier.

Unity3D: This is the development platform where the DS model and all driving scenes were developed, executed and a standalone created. Unity3D has an asset store which offers numerous free and commercial inbuilt models for easy integration. Furthermore, it also offers a convincing and near-realistic experience in the virtual world due to its excellent graphics. Unity5 provides almost accurate vehicle physics for an absolute realistic vehicle control. Finally, Unity3D supports force feedback devices e.g. Logitech G25. The two main functions implemented in Unity3D are the Camera Controller and the Vehicle Controller (Driving Script) where the vehicle dynamics are implemented. 


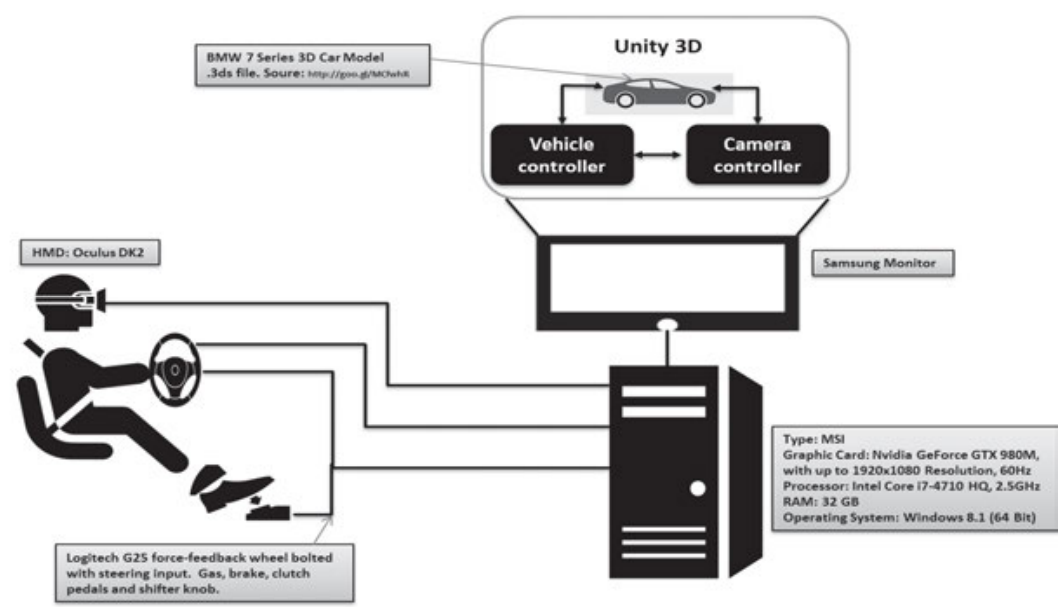

Figure 1: Graphical representation of the VR DS System Architecture with the user wearing the OR.

\section{Prototype Development and Evaluation}

\subsection{System Architecture}

For easy integration of new features, the design of the VR driving simulator followed a modular-based architecture. The design consisted of integrating the vehicle model in Unity 3D, simulating realistic lighting conditions within Unity3D, integration of the $3 \mathrm{D}$ models and input/out device mentioned in chapter 3 in Unity3D. Figure 1 shows the system architecture. OR with the head tracking sensor is connected to the computer through HDMI and USB interfaces. The HDMI is used to transmit the driving simulation graphic data to the HMD and the USB is used to control the head tracking sensor.

\subsection{Implementation}

The prototype was realized in Unity3D engine. The developed prototype was iteratively tested until the expected result was achieved. Implementation and testing were done in parallel, feature updates or corrections were tested in Unity3D with and without OR.

The next step was the integration of the male character model, the V8 engine sound, and the simulated driving speed displayed as a HuD feature. The HuD feature was implemented as a standard floating GUI text that displays the actual speed during runtime. Finally, the keyboard was configured to activate and deactivate the Logitech G25 functionality (F1), display the male character (driver) (F2) displayed in figure 2, and to display a motorbike driving directly opposite the test driver (F3) as shown in figure 3. 


\subsection{User Evaluation}

In order to validate the level of immersion and the near-realistic driving experience provided by the VR DS, and get user feedback on how to improve the implemented simulator, a user test with a selection of 25 users was performed.

The selected user evaluation methods consisted of a Pre-Test (PrTQ) and Post-Test (PoTQ) questionnaires. The PrTQ consisted of three sections namely personal data, experience with 3D gaming and HMD, and driving experience. This was filled before the test session in order to ascertain the user's vulnerability to experience motion sickness. Users who had little or no experience with 3D gaming or never worn a HMD before, were forewarned.

The first 14 out of the 20 questions of the PoTQ were based on expert evaluation from the IGroup Presence Questionnaire (IPQ) to determine Presence in VEs (iGroup, 2008). Content was mainly the user experience with the implemented prototype. The questionnaire was filled by test persons after the driving experience in order to assess if the driving experience with the DS was felt as real or not. The questions are mainly related to typical VR systems and their comparison with the real world.

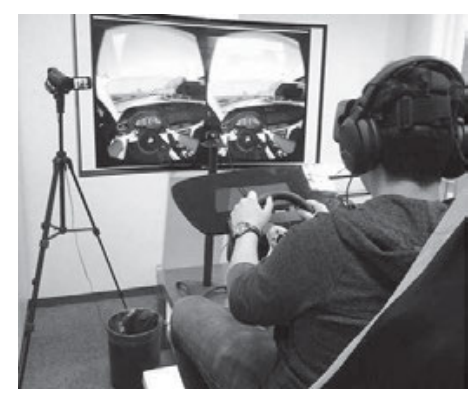

Figure 2: VR Simulator setup displaying the male character model during a during a test session

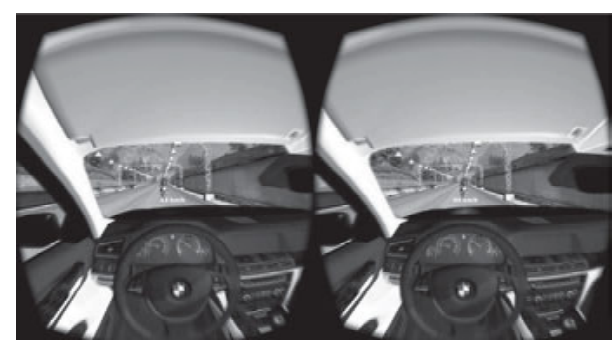

Figure 3: VR DS showing the sudden appearance of a motorcyclist in order to analyze the driver's reaction towards avoiding the head-on collision. 


\subsubsection{Users}

The selection of test drivers was made without any pre-information about the users. All users were Robert Bosch employees working in Leonberg. The only criteria stated for the selection is the availability of a driver's license though, more users with little or no VR experience and VE interactions were preferable in order to avoid biases. Two users however, did not have a driver's license. 20 out of the 25 users were male while 5 were female. The age ranged from 22 to 63 with an average of 37.6. 20 users had at least a university degree while the rest were students. Apart from two users, all have watched a 3D movie with regular 3D glasses. 20 users owned a driving license older than 5 years. An average duration of 30 minutes was planned for each user. See table 2 for the representation of four PrTQ answers.

\subsubsection{User test execution and test scenario}

Firstly, the PrTQ was filled out, the user was then explained the basic functionality of the simulator. The occurrence and symptoms of simulator sickness was also mentioned prior to the test proper. In order to capture all gestures during the session the video camera was turned on. Next, the user was wearing the OR DK2 and 5.1 Roccat KAVE surround earphones in order to experience the V8 engine sound and other surrounding sounds integrated in the system. While the test was ongoing, the scenario was manipulated with the MSI laptop by activating and deactivating the driver model and the motorbike scene. The driving task involved a typical driving maneuver including curve driving, backward driving, braking, as well as lane departure warning maneuver, reaction to the sudden appearance of the motorbike riding towards the test car in order to avoid a head-on collision (figure 2). After performing all driving tasks, the user finally was requested to fill the PoTQ.

\subsubsection{User test results}

The quantitative analysis of some of the responses of the PoTQ shows the following results; For Question (Q) 16, the users were asked if they did not feel present in the VE. 76\% of the users felt present, $16 \%$ did feel present and $8 \%$ were neutral. The result from Q11- I still paid attention to the real environment (RE) - shows that $72 \%$ did not pay attention to the RE, 20\% still paid attention and 2\% were neutral. Another interesting result is Q12- the VE seemed more realistic than the RE- $92 \%$ did not agree while $8 \%$ were neutral. $84 \%$ of the users felt motion sick during the tests. Most Simulation sickness (SS) occurred while driving through a sharp curve. Four out of the five female participants suffered from SS within the first five minutes of the test and 3 had to interrupt the session. All users with little or no experience with HMDs for except one, displayed most symptoms of motion sickness (Better Health Channel, 2015) e.g. sweating, increased salivation, nausea, malaise. However, there was no case of vomiting. 


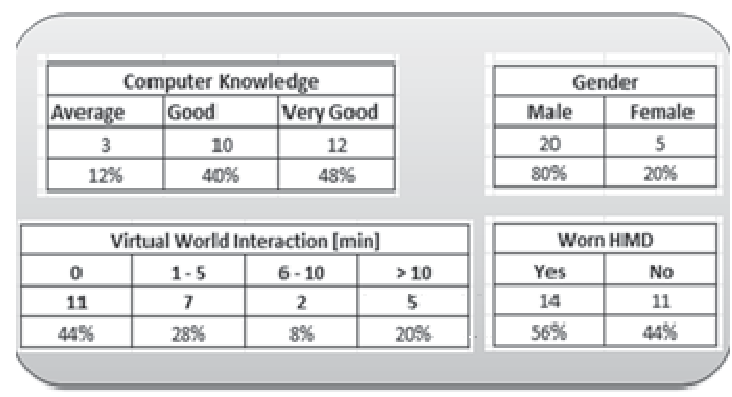

Table 2: User data on computer knowledge, gender, VE interaction and HMD.

\section{Discussion and Future Work}

This work focused on realizing 3D VR DS which compensates for the lack of total immersion experienced with 2D DSs by immersing the driver in the computer-generated driving environment. Hence the use of VR allows the user to "interact" with and become "immersed" in the computer-generated driving environment in a natural way (Schultheis \& Rizzo, 2001).

In order to realize the VR DS, suitable tools were selected, analyzed and evaluated through benchmarks. This phase played a very important role in the overall prototype implementation because a high performing and near-realistic VR application depends on the resolution, the level of immersion offered by the HMD and the driving simulation tool applied to implement and create the standalone application. The developed VR DS prototype implemented in Unity3D engine was validated through performing user tests with a total of 25 test drivers.

The Wheel Colliders and simple Physics offered by Unity 3D was not enough to simulate a realistic car model. The right feeling of speed was missing e.g. $100 \mathrm{~km} / \mathrm{h}$ felt like $20 \mathrm{~km} / \mathrm{h}$. Therefore, an object-oriented script was written to simulate a car's behavior. This issue persisted, but was better with the introduction of Unity5 which offered better Physics.

Though the VR DS felt immersive, its level of immersion was limited due to the occurrence SS caused most probably by a motionless simulator, poor graphical performance of the driving scenes, incorrect scaling of the perceived speed and acceleration as commented by most test drivers. It was observed during the user evaluation test, that the occurrence of SS limits the benefits of a VR experience by influencing the driver's performance during testing of new safety functions and HMI prototype evaluation. Most female test participants did not experience total immersion. Most male participants on the other hand were totally immersed in the VE. This may be due to the fact that most female participants had motion sickness quite early and hence had to stop the test before full immersion and total disconnection from the RE was reached. This also confirms the known theory of motion sickness occurrence during simulation and VR applications especially with motionless DSs, missing force feed- 
back and low resolution (Karl et al., 2013; Davis et al., 2014). By eliminating these limitations, the full potential of VR DS could be effectively applied to generate an efficient and near-realistic driving experience of hazardous driving situations without endangering the life of the driver (De Winter et al., 2012; Kemeny, 2014).

Another limitation of the system was that, the simulation of a realistic congested traffic model was missing. The BMW 7 series was the only vehicle in the VE, no pedestrians and traffic signs. Most users commented that it conflicted with the image they have of a real road. Therefore, it is essential to simulate a real traffic model with all related features if the VE is expected to be perceived as real.

Because of the benefits provided by a full VR solution, this work continues. The future work should focus on finding the causes and possible solutions of SS and the elimination of the challenges mentioned above. Planned is also the development of clickable interfaces for e.g. Infotainment system and Data glove integration for a better interaction. Finally, a 2D DS should be compared with a VR DS in order to ascertain the benefits of VR DS. The main objective would be the implementation and evaluation of an immersive and near-realistic high performance 3D VR DS for a better user experience that encourages the user-centered adaptations of driving safety functions and for rapid prototyping of new HMI concepts.

\section{Acknowledgment}

I would like to thank Jonas Roth for his assistance in the implementation of this work.

\section{Literaturverzeichnis}

Bayarri, S., Fernandez, M., \& Perez, M. (1996). Virtual reality for driving simulation. Communications of the ACM 39.5, P. 72-76.

Better Health Channel (2015). Motion Sickness. Website: http://goo.gl/BQxED8

Christodoulou, S., Despina, M., Gregoriades, A., \& Pampaka, M. (2013). Design of a 3D interactive simulator for driver behavior analysis. Summer Computer Simulation Conference (SCSC '13). Society for Modeling \& Simulation International, Vista, CA, Article 17, 8 pages.

Cremer, J., Kearney, J., \& Papelis, Y. (1996). Driving simulation: challenges for VR technology. Computer Graphics and Applications, IEEE 16.5: P.16-20.

De Winter, J.F.C, Van Leuween, P., \& Happee. R. (2012). Advantages and Disadvantages of Driving Simulators: A Discussion. In Proceedings of Measuring Behavior.

FurryBall 4.8 GPU Benchmark, (2014). The best graphics cards for raytrace in FurryBall. Website: http://goo.gl/9NMSwA

iGroup (2008). igroup presence questionnaire. Website: http://tinyurl.com/oll6d7z.

Karl, Ines, et al. (2013). Driving behavior and simulator sickness while driving the vehicle in the loop: Validation of longitudinal driving behavior. IEEE intelligent transportation systems magazine 5.1: P. 42-57.

Kemeny, A. (2014). From Driving Simulation to Virtual Reality. Center for Virtual Reality and Immersive Simulation. Paris: Renault. 
Krueger, H-P., et al. (2005). SILAB-A task-oriented driving simulation. Proceedings of the Driving Simulator Conference (DSC). Orlando.

Reddit (2014). Buying graphics cards for Oculus rift DK2. Website: http://goo.gl/aiGyfA

Robinson, S. (2004). Simulation: The Practice of Model Development and Use. Chichester: John Wiley and sons.

Schultheis, M.T., Rizzo, A.A. (2001). The application of virtual reality technology in rehabilitation. Rehabilitation Psychology. Vol 46(3): P. 296-311.

TopTenReviews (2015). Graphics Cards Review and comparisons. Website: http://tinyurl.com/6gdss71. 